\title{
Inseparable relationships between stem cells and the niche: a case study in Drosophila
}

Ting Xie ${ }^{1}$

${ }^{1}$ Stowers Institute for Medical Research, 1000 East 50th Street, Kansas City, MO 64110, USA

Stem cells in adult tissues have the capacity to self-renew and generate differentiated progeny that replenish lost or damaged cells. The Drosophila ovary is an effective system for studying relationships between the niche and stem cells because the stem cells and their associated niches are well defined and versatile genetic tools for dissecting gene functions are readily available. My lab focuses on two types of stem cells in the Drosophila ovary: germline stem cells (GSCs) for generating oocytes and follicle stem cells (FSCs) for producing somatic follicular epithelial cells. Using a combination of genetic and cell biological approaches, we have studied multiple signaling transduction pathways for their roles in controlling stem cell self-renewal and differentiation, such as BMP, Notch and Wnt. In addition, we have revealed essential roles of cadherin-mediated cell adhesion in anchoring GSCs and FSCs to their niches, and have defined essential functions of ATP-dependent chromatin remodeling factors and the microRNA pathway in controlling GSC and FSC self-renewal. Recently, we have shown that Notch signaling is required for GSC niche formation and maintenance. In this meeting, I will report our recent progress in understanding of adult stem cell regulation in the Drosophila ovary.

Keywords: stem cell, self-renewal, niche, germline

Cell Research (2008) 18:s93. doi: 10.1038/cr.2008.183; published online 4 August 2008

Correspondence: Ting Xie

E-mail: tgx@Stowers-Institute.org

Ting Xie is an associate investigator of Stowers Institute for Medical Research and an associate professor of Anatomy and Cell Biology at University of Kansas Medical Center. Dr Xie received his PhD. degree in Biochemistry and Molecular Biology from Rutgers University, working with Dr Richard Padgett at Waksman Institute. He was a postdoctoral fellow with Dr Allan Spradling at Howard Hughes Medical Institute and Carnegie Institution of Washington. His laboratory studies on the molecular mechanisms underlying stem cell self-renewal, proliferation and differentiation in Drosophila and mice using a combination of genetics, molecular biology, biochemistry, genomics and cell biology. 